\title{
THE LOCAL BEHAVIOR OF PRINCIPAL AND CHORDAL PRINCIPAL CLUSTER SETS
}

\author{
BY \\ JOHN T. GRESSER
}

\begin{abstract}
Let $K$ be the unit circle, and let $f$ be a function whose domain is the open unit disk and whose range is a subset of the Riemann sphere. We define a set, called the boundary principal cluster set of $f$ at $\zeta_{0} \in K$, which characterizes the behavior of the principal cluster sets of $f$ at points $\zeta \in K$ which are near $\zeta_{0}$ and distinct from $\zeta_{0}$. It is shown that if $f$ is continuous, then the principal and boundary principal cluster sets of $f$ at $\zeta_{0}$ are equal for nearly every point $\zeta_{0} \in K$. A similar result holds for chordal principal cluster sets. Examples are provided that indicate directions in which the result cannot be improved. Some results concerning points that are accessible through sets which are unions of arcs are also presented.
\end{abstract}

1. Introduction: Definitions and notation. Let $D$ be the open unit disk, and let $K$ be the unit circle. We say that $\alpha$ is an arc at $\zeta_{0} \in K$ if $\alpha$ is contained in $D$, and is the image of a continuous function $z=z(t)(0 \leqq t<1)$ such that $z(t) \rightarrow \zeta_{0}$ as $t \rightarrow 1$. We say that $\beta$ is a terminal subarc of $\alpha$ if $\beta=z\left(\left[t_{0}, 1\right)\right)$ for some $t_{0}\left(0 \leqq t_{0}<1\right)$ and some representative $z=z(t)$ of $\alpha$. If $\mathrm{P}$ is a property which is meaningful for each point in $K$, then we say that nearly every point in $K$ has property $\mathrm{P}$ if the exceptional set is a set of first Baire category in $K$. It is assumed that the reader is familiar with some of the elementary notions of cluster set theory (see [4] or [6]). Thus if $f$ is a function whose domain is $D$ and whose range is a subset of the Riemann sphere, if $\zeta_{0} \in K$ and if $\alpha$ is an arc at $\zeta_{0}$, then $C\left(f, \zeta_{0}, \alpha\right)$ denotes the arc cluster set of $f$ at $\zeta_{0}$ along $\alpha$.

We will approach our study of principal and chordal principal cluster sets through the following more general concept. We call $\Gamma$ a selector of $\operatorname{arcs}$ if $\Gamma$ is a correspondence which associates with each $\zeta \in K$ a nonempty collection $\Gamma(\zeta)$ of arcs at $\zeta$. If $\Gamma$ is a selector of arcs, we define the $\Gamma$-principal cluster set of $f$ at $\zeta$ to be the set

$$
\Pi_{\Gamma}(f, \zeta)=\bigcap_{\alpha} C(f, \zeta, \alpha)
$$

where $\alpha$ ranges over all arcs in $\Gamma(\zeta)$. If for each $\zeta \in K, \Gamma(\zeta)$ is the collection of all arcs at $\zeta$, then the corresponding set $\Pi_{\Gamma}(f, \zeta)$ is called the principal cluster set of $f$ at $\zeta$ and it is denoted by $\Pi(f, \zeta)$. The chordal principal cluster set of $f$ at $\zeta$ is the set $\Pi_{x}(f, \zeta)$ where $\chi(\zeta)$ is the collection of all chords at $\zeta(\zeta \in K)$.

Received by the editors February 4, 1971 and, in revised form, July 14, 1971.

AMS 1970 subject classifications. Primary 30A72; Secondary 30A90.

Key words and phrases. Principal cluster set, chordal principal cluster set, accessible points, Baire category, three-arc property. 
The major purpose of this article is to show that the well-known result of E. F. Collingwood concerning boundary cluster sets (see [4, p. 82]) can, with some modifications, be formulated in the context of $\Gamma$-principal cluster sets for some choices of $\Gamma$, including those which yield the principal and chordal principal cluster sets. For any positive number $\mu$, and for any $\zeta_{0} \in K$, let $K_{\mu}$ denote the set

$$
K_{u}=\left\{\zeta \in K: 0<\left|\zeta-\zeta_{0}\right|<\mu\right\} .
$$

Then if $f$ is defined in $D$, and $\Gamma$ is a selector of arcs, we define

$$
\Pi_{\Gamma}\left(f, \zeta_{0}, \mu\right)=\bigcup_{\zeta} \Pi_{\Gamma}(f, \zeta),
$$

where $\zeta$ ranges over all points in $K_{\mu}$. We then let $\Pi_{\Gamma}^{*}\left(f, \zeta_{0}, \mu\right)$ denote the closure of $\Pi_{\Gamma}\left(f, \zeta_{0}, \mu\right)$ in the Riemann sphere. The boundary $\Gamma$-principal cluster set of $f$ at $\zeta_{0}$ is defined to be the set

$$
B \Pi_{\Gamma}\left(f, \zeta_{0}\right)=\bigcap_{\mu>0} \Pi_{\Gamma}^{*}\left(f, \zeta_{0}, \mu\right)
$$

We will show that if $f$ is continuous, then $B \Pi_{\Gamma}\left(f, \zeta_{0}\right)=\Pi_{\Gamma}\left(f, \zeta_{0}\right)$ nearly everywhere in $K$ provided that $\Gamma$ is an appropriate selector of arcs.

Let $\Gamma$ be a selector of arcs, and let $S \subseteq D$. We say that a point $\zeta_{0} \in K$ is almost $\Gamma$-accessible through $S$ if for every open set $G$ with $S \subseteq G \subseteq D$ there is an arc $\alpha \in \Gamma\left(\zeta_{0}\right)$ such that $\alpha \subseteq G$. If $\Gamma\left(\zeta_{0}\right)$ is the collection of all arcs at $\zeta_{0}$, if $S \subseteq D$, and if $\zeta_{0}$ is almost $\Gamma$-accessible through $S$, then we say that $\zeta_{0}$ is almost accessible through $S$. Now let $E \subseteq K$, and let $\gamma$ be a correspondence which associates with each $\zeta \in E$ an $\operatorname{arc} \gamma(\zeta) \in \Gamma(\zeta)$. We let $\bar{S}(\gamma, E)$ be the relative closure in $D$ of the set

$$
S(\gamma, E)=\bigcup_{\zeta \in E} \gamma(\zeta)
$$

Then we say that $\Gamma$ is a smooth selector of arcs if for every set $E$ of second category in $K$, and every such $\gamma$, there is a subarc $A \subseteq K$ such that $E$ is dense in $A$ and each point of $A$ is almost $\Gamma$-accessible through $\bar{S}(\gamma, E)$. If $\Gamma$ is a selector of arcs, we define a new selector of arcs $\Gamma^{\prime}$, called the completion of $\Gamma$, by the expression

$$
\Gamma^{\prime}(\zeta)=\{\alpha: \alpha \text { is a terminal subarc of an } \operatorname{arc} \beta \in \Gamma(\zeta)\} \quad(\zeta \in K) .
$$

Finally we say that $\Gamma$ is an admissible selector of arcs if the completion of $\Gamma$ is a smooth selector of arcs.

2. The geometry of arcs. In order to establish our major results concerning principal cluster sets we will need to establish that certain selectors of arcs are admissible. Our first result in that direction concerns the classic notion of accessibility.

Lemma 1. Let $A$ be an open subarc of the unit circle, let $E \subseteq K$ be dense in $A$, and let $\rho$ be a fixed real number with $0 \leqq \rho<1$. Let $\gamma$ be a correspondence which associates 
with each $\zeta \in E$ an arc $\gamma(\zeta)$ at $\zeta$ which intersects the circle $|z|=\rho$. Then each point of $A$ is almost accessible through $\bar{S}(\gamma, E)\left({ }^{1}\right)$.

Proof. Let $\bar{S}=\bar{S}(\gamma, E)$ and let $G$ be an open set such that $\bar{S} \subseteq G \subseteq D$. We will be using the notion and subsequent properties of convergence of a sequence of sets to a limit set as described in [7, pp. 10-15]. In this proof the terms convergence and limit of a sequence of arcs should be understood in this context.

Let $\zeta_{0} \in A$, and let $D_{0}$ be an open disk centered at $\zeta_{0}$ having radius $r \leqq 1-\rho$. Let $\left\langle\zeta_{j}\right\rangle$ be a sequence of distinct points in $E \cap D_{0}$ which converges to $\zeta_{0}$. Then for each $j$, let $\gamma_{j}$ be the component of $\gamma\left(\zeta_{j}\right) \cap D_{0}$ which forms a terminal subarc of $\gamma\left(\zeta_{j}\right)$. We assert that there is a component $G_{0}$ of $G \cap D_{0}$ such that

$$
\gamma_{j} \subseteq G_{0} \text { for infinitely many values } j \text {. }
$$

To prove this let $\left\langle\gamma_{j_{k}}\right\rangle$ be a subsequence of $\left\langle\gamma_{j}\right\rangle$ which converges to a limit set $L$, and for convenience set $\alpha_{k}=\gamma_{j_{k}}$. In the case that $L \cap D_{0} \cap D \neq \varnothing$, let $z \in L \cap D_{0}$ $\cap D$. Then $z \in \bar{S} \cap D_{0}$ so that $z$ is contained in some component $G_{0}$ of $G \cap D_{0}$. Since $z \in L \cap G_{0}$ and $G_{0}$ is open, it follows from the definition of limit that there is an $N$ such that $G_{0} \cap \alpha_{k} \neq \varnothing$ for all $k>N$. Thus since $\alpha_{k}$ is a connected subset of $G \cap D_{0}$ it follows that $\alpha_{k} \subseteq G_{0}$ for all $k>N$. This proves the assertion in the case that $L \cap D_{0} \cap D=\varnothing$. In the case that $L \cap D_{0} \cap D=\varnothing$ we use the elementary properties of convergence in [7, pp. 10,15] to conclude that $L \cap K$ is a nondegenerate continuum. Now let $\zeta \in L \cap E \cap D_{0}$ and let $\alpha$ be the component of $\gamma(\zeta) \cap D_{0}$ which forms a terminal subarc of $\gamma(\zeta)$. Then using the definition of convergence, and the Jordan Curve Theorem, it easily follows that there is an $N$ such that $\alpha \cap \alpha_{k} \neq \varnothing$ for all $k>N$. Since $\alpha$ is a connected subset of $G \cap D_{0}$, it follows that $\alpha$ is contained in a component $G_{0}$ of $G \cap D_{0}$. Since $\alpha_{k}$ is a connected subset of $G \cap D_{0}$ we conclude that $\alpha_{k} \subseteq G_{0}$ for all $k>N$. This proves the assertion in the remaining case.

Let $D_{k}$ be the open disk centered at $\zeta_{0}$ having radius $(1-\rho) / k(k=1,2,3, \ldots)$. We use (1) to construct a sequence $\left\langle G_{k}\right\rangle$ of open connected sets such that

$$
G \supseteq G_{1} \supseteq G_{2} \supseteq G_{3} \supseteq \cdots, \text { and } G_{k} \subseteq D_{k} \quad(k=1,2,3, \ldots) .
$$

Our construction is by induction and it requires, in addition, the construction of certain collections of arcs. For this purpose we introduce the following notation. Let $\alpha$ be an arc at $\zeta \in E$. If $\zeta \in D_{k}$ we let $\alpha^{k}$ be the component of $\alpha \cap D_{k}$ which forms a terminal subarc of $\alpha$. If $\zeta \notin D_{k}$ we let $\alpha^{k}=\varnothing$. Now let $\left\langle\zeta_{j}\right\rangle$ be a sequence of distinct points in $E$ which converges to $\zeta_{0}$. Using (1) we select an infinite subset $T_{1}$ of $\left\{\gamma\left(\zeta_{j}\right)\right\}$, and a component $G_{1}$ of $G \cap D_{1}$ such that

$$
\varnothing \neq \alpha^{1} \subseteq G_{1} \text { for every } \alpha \in T_{1} .
$$

(1) The author shows in a forthcoming article that it is possible to have a dense set of nonaccessible points of $\bar{S}(\gamma, E)$ even when $E$ contains nearly every point of $K$. 
Using (1) again we select an infinite subset $T_{2}$ of $T_{1}$ and a component $G_{2}$ of $G \cap D_{2}$ such that

$$
\varnothing \neq \alpha^{2} \subseteq G_{2} \text { for every } \alpha \in T_{2} .
$$

This process can clearly be continued inductively so as to define sequences $\left\langle G_{k}\right\rangle$ and $\left\langle T_{k}\right\rangle$ such that for each $k=1,2,3, \ldots$,

$G_{k}$ is a component of $G \cap D_{k}$,

$\left\{\gamma\left(\zeta_{j}\right)\right\} \supseteq T_{1} \supseteq T_{2} \supseteq T_{3} \supseteq \cdots$,

$\varnothing \neq \alpha^{k} \subseteq G_{k}$ for every $\alpha \in T_{k}$.

To show that the sequence $\left\langle G_{k}\right\rangle$ satisfies (2), we let $n$ be fixed and let $\alpha \in T_{n+1} \subseteq T_{n}$. Then since $\varnothing \neq \alpha^{n+1} \subseteq \alpha^{n}$, and $\alpha^{n+1} \subseteq G_{n+1}$, and $\alpha^{n} \subseteq G_{n}$, it follows that $G_{n+1} \cap G_{n}$ $\neq \varnothing$. But $G_{n+1} \subseteq G \cap D_{n+1} \subseteq G \cap D_{n}$, so that since $G_{n+1}$ is connected and $G_{n}$ is a component of $G \cap D_{n}$ which intersects $G_{n+1}$, we may conclude that $G_{n+1} \subseteq G_{n}$.

Using the sequence $\left\langle G_{k}\right\rangle$ just constructed and the arcwise connectedness of each $G_{k}$, it is now easy to construct an arc at $\zeta_{0}$ which lies in $G$.

By a closed Stolz angle at a point $\zeta_{0} \in K$ we mean a Stolz angle at $\zeta_{0}$ which contains all its boundary points except $\zeta_{0}$. Let $\Delta$ be a closed Stolz angle at $\zeta_{0}=1$ and let $\Delta(\zeta)$ be the closed Stolz angle at $\zeta$ obtained by a rotation of $\Delta$ about the origin.

Lemma 2. Let $A$ be an open subarc of $K$, let $E \subseteq K$ be dense in $A$, and let $\rho$ be a fixed real number with $0 \leqq \rho<1$. Let $\gamma$ be a correspondence which associates with each $\zeta \in E$ a chord $\gamma(\zeta)$ at $\zeta$ which lies in $\Delta(\zeta)$, and intersects the circle $|z|=\rho$. Then for each $\zeta \in A$ there is a chord at $\zeta$ which lies in both $\Delta(\zeta)$ and in $\bar{S}(\gamma, E)$.

Proof. The proof of this lemma is immediate. Let $\zeta_{0} \in A$, and let $\left\langle\zeta_{j}\right\rangle$ be a sequence of points in $E$ which converges to $\zeta_{0}$. As a sequence of sets, the sequence $\left\langle\gamma\left(\zeta_{j}\right)\right\rangle$ has a subsequence which converges to a set $L$. The set $L$ is clearly a chord at $\zeta_{0}$ which lies in both $\Delta\left(\zeta_{0}\right)$ and in $\bar{S}(\gamma, E)$.

For each $\zeta \in K$ let $\Lambda(\zeta)$ be the collection of all arcs at $\zeta$ and let $\chi_{\Delta}(\zeta)$ be the collection of all chords at $\zeta$ which are contained in $\Delta(\zeta)$.

THEOREM 1. Each of the correspondences $\Lambda$ and $\chi_{\Delta}$ is an admissible selector of arcs.

Proof. We prove this only for $\Lambda$ since the argument is the same for $\chi_{\Delta}$. Let $E$ be a second category subset of $K$, and for each $\zeta \in E$ let $\gamma(\zeta) \in \Lambda(\zeta)$. For each $j=1,2,3$, ... define the set

$$
E_{j}=\{\zeta \in E: \gamma(\zeta) \text { intersects the circle }|z|=1-1 / j\} .
$$

Then $E$ is the union of the sets $E_{j}(j=1,2,3, \ldots)$ so that there is an $N$ such that $E_{N}$ is of second category in $K$. Therefore there is an open subarc $A \subseteq K$ such that $E_{N}$ is dense in $A$. The proof is completed by applying Lemma 1 to the set $E_{N}$.

3. The major results. Our first result depends only on the topology of closed subsets of a compact space, and consequently it is stated in a more general setting. 
THEOREM 2. Let $f$ be an arbitrary complex valued function defined in $D$, and let $\Gamma$ be a selector of arcs. Then $\Pi_{\Gamma}(f, \zeta) \subseteq B \Pi_{\Gamma}(f, \zeta)$ for all but at most a countable number of points $\zeta \in K$.

Proof. For each $j=1,2,3, \ldots$ let $\mathfrak{T}_{j}$ be a finite collection of compact neighborhoods of the Riemann sphere $W$ which cover $W$ and such that, in terms of the usual metric for $W$, dia $(G)<1 / j$ for each $G \in \mathfrak{T}_{j}$. We index the collection $\mathfrak{T}=\bigcup_{j=1}^{\infty} \mathfrak{T}_{j}$ in any convenient manner and denote this collection by $\mathfrak{T}=\left\{G_{j}\right\}$. Now define the set

and the sets

$$
P=\left\{\zeta \in K: \Pi_{\Gamma}(f, \zeta) \nsubseteq B \Pi_{\Gamma}(f, \zeta)\right\},
$$

$$
P_{j}=\left\{\zeta \in P: G_{j} \cap \Pi_{\Gamma}(f, \zeta) \neq \varnothing \text { and } G_{j} \subseteq W-B \Pi_{\Gamma}(f, \zeta)\right\} \quad(j=1,2,3, \ldots) .
$$

If $\zeta \in P$, then there is a point $w \in \Pi_{\Gamma}(f, \zeta)$ such that $w \notin B \Pi_{\Gamma}(f, \zeta)$. Since $B \Pi_{\Gamma}(f, \zeta)$ is a closed subset of $W$, it follows that there is a $k$ such that $w \in G_{k}$ and $G_{k} \cap B \Pi_{\Gamma}(f, \zeta)=\varnothing$. This implies that $\zeta \in P_{k}$, and we have proved that $P$ is the union of the sets $P_{j}(j=1,2,3, \ldots)$. Thus it suffices to show that each of the sets $P_{j}$ is at most countable. To prove this we fix $j$ and let $\zeta \in P_{j}$. We assert that $\zeta$ is an isolated point of $P_{j}$, for if not, there is a sequence $\left\langle\zeta_{k}\right\rangle$ of points in $P_{j}$ which converges to $\zeta$. Since each $\zeta_{k} \in P_{j}$ it follows that $G_{j} \cap \Pi_{\Gamma}\left(f, \zeta_{k}\right) \neq \varnothing(k=1,2,3, \ldots)$. Thus for each $\mu>0$

$$
G_{j} \cap \Pi_{\Gamma}^{*}(f, \zeta, \mu) \neq \varnothing .
$$

We let $\left\langle\mu_{k}\right\rangle$ be a decreasing sequence of positive real numbers which converges to zero, and write

$$
G_{j} \cap B \Pi_{\Gamma}(f, \zeta)=\bigcap_{k=1}^{\infty}\left(G_{j} \cap \Pi_{\Gamma}^{*}\left(f, \zeta, \mu_{k}\right)\right) .
$$

In view of (3) and the compactness of $W$ we conclude that $G_{j} \cap B \Pi_{\Gamma}(f, \zeta) \neq \varnothing$, and this contradicts the assumption that $\zeta \in P_{j}$. Thus each $\zeta \in P_{j}$ is an isolated point and the theorem is proved.

The next lemma is a trivial application of the definition of $\Pi_{\Gamma}(f, \zeta)$ and so its proof will be omitted.

Lemma 3. Let $f$ be defined in $D$, and let $\Gamma$ be a selector of arcs. Let $G$ be any open subset of the Riemann sphere such that, for some $\zeta \in K, G \cap B \Pi_{\Gamma}(f, \zeta) \neq \varnothing$. Then there is a sequence $\left\langle\zeta_{j}\right\rangle$ of points in $K$ which converges to $\zeta$ such that $G \cap \Pi_{\Gamma}\left(f, \zeta_{j}\right) \neq \varnothing$ for every $j$.

LEMMA 4. Let $f$ be continuous in $D$, and let $\Gamma$ be an admissible selector of arcs. For each $\zeta \in K$ let $\beta_{\zeta} \in \Gamma(\zeta)$. Then $B \Pi_{\Gamma}(f, \zeta) \subseteq C\left(f, \zeta, \beta_{\zeta}\right)$ for nearly every point in $K$.

Proof. Suppose that the lemma is false, so that the set

$$
P=\left\{\zeta \in K: B \Pi_{\Gamma}(f, \zeta) \ddagger C\left(f, \zeta, \beta_{\zeta}\right)\right\}
$$


is of second category in $K$. In several steps we extract a subset of $P$ which is also of second category in $K$.

For any given $\varepsilon>0$ and $\zeta \in K$, let $C(\zeta, \varepsilon)$ denote the set of all points in the Riemann sphere $W$ whose spherical distance from $C\left(f, \zeta, \beta_{\zeta}\right)$ does not exceed $\varepsilon$. For any $\zeta \in P$, since $C\left(f, \zeta, \beta_{\zeta}\right)$ is a closed set in $W$, it follows that there is an $\varepsilon>0$ such that $B \Pi_{\Gamma}(f, \zeta)-C(\zeta, \varepsilon) \neq \varnothing$. Let $\left\langle\varepsilon_{j}\right\rangle$ be a decreasing sequence of positive numbers which converges to zero, and define the sets

$$
P(j)=\left\{\zeta \in P: B \Pi(f, \zeta)-C\left(\zeta, \varepsilon_{j}\right) \neq \varnothing\right\} \quad(j=1,2,3, \ldots) .
$$

We clearly have that $P$ is the union of the sets $P(j)(j=1,2,3, \ldots)$, so that since $P$ is of second category there is a $J$ such that $P(J)$ is of second category.

We now cover the Riemann sphere with a finite collection $\left\{G_{1}, G_{2}, \ldots, G_{m}\right\}$ of open sets each of diameter less than $\varepsilon_{J} / 4$. We define the sets

$$
P(J, \mu)=\left\{\zeta \in P(J): G_{\mu} \cap\left(B \Pi_{\Gamma}(f, \zeta)-C\left(\zeta, \varepsilon_{J}\right)\right) \neq \varnothing\right\} \quad(\mu \leqq m) .
$$

Since $P(J)$ is the union of the sets $P(J, \mu)(\mu \leqq m)$ it follows that there is an $M$ such that $P(J, M)$ is of second category.

Let $A, B$ be subsets of the Riemann sphere. For any $a \in A$ and $b \in B$ we let $\delta(a, b)$ denote the spherical distance between $a$ and $b$, and define

$$
\delta(A, B)=\inf \{\delta(a, b): a \in A, b \in B\} .
$$

With this notation it is easy to prove from (4) that

$$
\delta\left(G_{M}, C\left(f, \zeta, \beta_{\zeta}\right)\right) \geqq 3 \varepsilon_{J} / 4 \quad(\zeta \in P(J, M)) .
$$

Using (4) again we have that $G_{M} \cap B \Pi_{\Gamma}(f, \zeta) \neq \varnothing$ for each $\zeta \in P(J, M)$ so that by Lemma 3 it follows that every point $\zeta \in P(J, M)$ is a limit point of the set

$$
Q=\left\{\zeta \in K: G_{M} \cap \Pi_{\Gamma}(f, \zeta) \neq \varnothing\right\} .
$$

The remainder of the proof is devoted to showing that since (5) holds on a set of second category, it must hold on a full subarc of $K$ and this violates (6). To prove this we will make use of the definition and associated notation for an admissible selector of arcs. We let $E=P(J, M)$. Using the definition of an arc-cluster set, for each $\zeta \in E$ we let $\gamma(\zeta)$ be a terminal subarc of $\beta_{\zeta}$ such that

$$
f(\gamma(\zeta)) \subseteq C\left(\zeta, \varepsilon_{J} / 4\right) \quad(\zeta \in E)
$$

Then using the notation $S(\gamma, E)$ we have

$$
\bigcup_{\zeta \in E} f(\gamma(\zeta))=f\left(\bigcup_{\zeta \in E} \gamma(\zeta)\right)=f(S(\gamma, E)) \text {. }
$$

Therefore using (5), (7) it follows that $\delta\left(G_{M}, f(S(\gamma, E))\right) \geqq \varepsilon_{J} / 2$. Since $f$ is continuous it follows that $\delta\left(G_{M}, f(\bar{S}(\gamma, E))\right) \geqq \varepsilon_{J} / 2$. Let $G$ be an open set such that

$$
f(\bar{S}(\gamma, E)) \subseteq G \text { and } \delta\left(G_{M}, G\right) \geqq \varepsilon_{J} / 4 .
$$


Then since $f$ is continuous the set $U=f^{-1}(G)$ is an open subset of $D$ which contains $\bar{S}(\gamma, E)$. Since $\Gamma$ is an admissible selector of arcs, it follows that there is a subarc $A \subseteq K$ such that each point of $A$ is almost $\Gamma^{\prime}$-accessible through $\bar{S}(\gamma, E)$, where $\Gamma^{\prime}$ denotes the completion of $\Gamma$. Thus for every $\zeta \in A$ there is an arc $\alpha_{\zeta} \in \Gamma^{\prime}(\zeta)$ such that $\alpha_{\zeta} \subseteq U(\zeta \in A)$. Using the definition of $U$ we have

$$
C\left(f, \zeta, \alpha_{\zeta}\right) \subseteq \bar{G} \quad(\zeta \in A) .
$$

But $\alpha_{\zeta}$ is a terminal subarc of an arc in $\Gamma(\zeta)$ and as such the two corresponding arc-cluster sets are the same. Hence it follows from (9) that $\Pi_{\Gamma}(f, \zeta) \subseteq \bar{G}(\zeta \in A)$, so that by (8) we have

$$
\delta\left(G_{M}, \Pi_{\Gamma}(f, \zeta)\right) \geqq \varepsilon_{J} / 4 \quad(\zeta \in A) .
$$

The proof is completed by noting that (6) and (10) are untenable.

THEOREM 3. Let $f$ be an arbitrary complex-valued function defined in $D$, and let $\Gamma$ be any selector of arcs. Then there exists a selector of arcs $\Gamma_{0}$ such that, for each $\zeta \in K, \Gamma_{0}(\zeta)$ is a finite or countable subset of $\Gamma(\zeta)$ and $\Pi_{\Gamma}(f, \zeta)=\Pi_{\Gamma_{0}}(f, \zeta)(\zeta \in K)$.

Proof. The proof is identical to that of [5, Theorem 2, p. 260].

THEOREM 4. Let $f$ be a continuous function in $D$, and let $\Gamma$ be an admissible selector of arcs. Then $\Pi_{\Gamma}(f, \zeta)=B \Pi_{\Gamma}(f, \zeta)$ for nearly every point $\zeta \in K$.

Proof. According to Theorem 3, for each $\zeta \in K$ there is a finite or countable subset of $\Gamma(\zeta)$, say $\left\{\alpha_{j}(\zeta)\right\}$, such that $\Pi_{\Gamma}(f, \zeta)=\bigcap_{j=1}^{\infty} C\left(f, \zeta, \alpha_{j}(\zeta)\right)$. In the case that the set $\left\{\alpha_{j}(\zeta)\right\}$ is finite, we have, for the sake of convenience, taken the liberty of repeating one of the arcs infinitely often. For each $j$, let $P_{j}$ be the set

$$
P_{j}=\left\{\zeta \in K: B \Pi_{\Gamma}(f, \zeta) \neq C\left(f, \zeta, \alpha_{j}(\zeta)\right)\right\} .
$$

By Lemma 4, each of the sets $P_{j}$ is of first category in $K$. Therefore the set $P=\bigcup_{j=1}^{\infty} P_{j}$ is of first category in $K$. It easily follows that

$$
B \Pi_{\Gamma}(f, \zeta) \subseteq \Pi_{\Gamma}(f, \zeta) \quad(\zeta \in K-P) .
$$

The proof is completed by using Theorem 2 .

THEOREM 5. Let $f$ be a continuous function in $D$. Then $\Pi(f, \zeta)=B \Pi(f, \zeta)$ and $\Pi_{x}(f, \zeta)=B \Pi_{x}(f, \zeta)$ for nearly every point $\zeta \in K$.

Proof. In view of Theorem 1 and Theorem 4 we need only prove this for the chordal principal cluster set. Let $\left\{\Delta_{j}\right\}$ be a countable collection of closed Stolz angles at $\zeta_{0}=1$ such that each chord at $\zeta_{0}=1$ is contained in at least one of the sets $\Delta_{j}(j=1,2,3, \ldots)$. For each $j$, let $\Delta_{j}(\zeta)$ be the closed Stolz angle at $\zeta \in K$ obtained by rotating $\Delta_{j}$ about the origin. Then for each $j$ and each $\zeta \in K$, let $\chi_{j}(\zeta)$ be the collection of all chords at $\zeta$ which are contained in $\Delta_{j}(\zeta)$. According to Theorem 1 and Theorem 4 , for each $j$ there is a set $E_{j}$ of first category in $K$ such that

$$
B \Pi_{x_{j}}(f, \zeta)=\Pi_{x_{j}}(f, \zeta) \quad\left(\zeta \in K-E_{j}\right) .
$$


Since $B \Pi_{x}(f, \zeta) \subseteq B \Pi_{x_{j}}(f, \zeta)$ for every $j$ and $\zeta \in K$ we have $B \Pi_{x}(f, \zeta) \subseteq \Pi_{x_{j}}(f, \zeta)$ $\left(\zeta \in K-E_{j}\right)$. The set $E=\bigcup_{j=1}^{\infty} E_{j}$ is of first category in $K$ and we have $B \Pi_{\chi}(f, \zeta)$ $\subseteq \Pi_{x_{j}}(f, \zeta)(\zeta \in K-E ; j=1,2,3, \ldots)$. Therefore

$$
B \Pi_{x}(f, \zeta) \subseteq \bigcap_{j=1}^{\infty} \Pi_{x_{j}}(f, \zeta)=\Pi_{x}(f, \zeta) \quad(\zeta \in K-E)
$$

and, in view of Theorem 2, the proof is complete.

The next corollary is in response to the following open question which has appeared in the literature (see [2, p. 14, Remark 2]). Does there exist a continuous function $f$ for which $\Pi_{x}(f, \zeta)=\varnothing$ for every point $\zeta$ in a set of second category in $K$ ? Although the corollary does not answer the question, it does provide some insight.

COROLlary 1. Let $f$ be a continuous function in $D$, and let $E \subseteq K$ be the set of all points $\zeta$ such that $\Pi_{\chi}(f, \zeta)=\varnothing$. Then if $E$ is of second category on $K$, it must contain a nondegenerate subarc of $K$.

Proof. It easily follows from the definition that if $f$ is any function, $\Gamma$ is any selector of arcs and $B \Pi_{\Gamma}\left(f, \zeta_{0}\right)=\varnothing$ for some point $\zeta_{0} \in K$, then $\Pi_{\Gamma}(f, \zeta)=\varnothing$ for all $\zeta$ in some nondegenerate subarc of $K$. The corollary now follows easily from Theorem 5.

Clearly the above corollary can be restated for any admissible selector of arcs. We show next that it can be restated in terms of another related topic. If $f$ is defined in $D$ and $\zeta \in K$, we say that $f$ has the three-arc property at $\zeta$ if there are three arcs at $\zeta$ such that the intersection of the three associated arc-cluster sets is empty.

COROLlaRY 2. Let $f$ be a meromorphic function in $D$. If $f$ has the three-arc property at each point of a set of second category in $K$, then $f$ has the three-arc property at each point of a nondegenerate subarc of $K$.

Proof. If $f$ has the three-arc property at a point $\zeta \in K$, then $\Pi(f, \zeta)=\varnothing$. If there is a set $E$ of second category of such points, it follows from Theorem 5 that for some point $\zeta_{0} \in K$ we must have $B \Pi\left(f, \zeta_{0}\right)=\varnothing$. Consequently just as in the proof of Corollary 1 , it then follows that $\Pi(f, \zeta)=\varnothing$ for each point $\zeta$ in some nondegenerate subarc $A$ of $K$. Then according to [3, Theorem 7, p. 220] $f$ has the three-arc property at each point of $A$.

4. Examples. The next two theorems indicate the sense in which Theorem 5 cannot be improved.

THEOREM 6. There exists a function $f$ defined in $D$ having a range of two values such that $\Pi(f, \zeta) \neq B \Pi(f, \zeta)$ and $\Pi_{x}(f, \zeta) \neq B \Pi_{x}(f, \zeta)(\zeta \in K)$.

Proof. Let $E$ be a countable dense subset of $K$, and let $F=K-E$. For each $\zeta \in E$, let $\rho_{\zeta}$ be the half open line segment having its closed end at the origin, and 
its open end at $\zeta$. We set

$$
P=\bigcup_{\zeta \in E} \rho_{\zeta}, \quad Q=D-P,
$$

and for each $z \in D$ define

$$
\begin{aligned}
f(z)=0 & \text { if } z \in P, \\
=1 & \text { if } z \in Q .
\end{aligned}
$$

It is easy to show that $\Pi(f, \zeta)=\Pi_{x}(f, \zeta)=\{0\}(\zeta \in E)$, and $\Pi(f, \zeta)=\Pi_{x}(f, \zeta)=\{1\}$ $(\zeta \in F)$. Since $E$ and $F$ are both dense in $K$, it follows that

$$
B \Pi(f, \zeta)=B \Pi_{x}(f, \zeta)=\{0,1\} \quad(\zeta \in K) .
$$

A function $f$ defined in $D$ is said to have the three-segment property at a point $\zeta \in K$ if there are three chords at $\zeta$ such that the intersection of the three associated arc-cluster sets is empty. This property is referred to in our next result.

THEOREM 7. There exists a continuous function $f$ defined in $D$ such that $B \Pi(f, \zeta)$ $\neq \Pi(f, \zeta)$ and $B \Pi_{\chi}(f, \zeta) \neq \Pi_{\chi}(f, \zeta)$ for every point of a perfect subset of $K$.

Proof. We refer to the function constructed in [1, Theorem 2, p. 30], and merely point out that the function referred to could have been improved in a trivial way to read as follows. There exists a continuous function $f$ in $D$ which has the threesegment property at each point of a perfect nowhere dense subset $E \subseteq K$ such that $f$ can be continuously extended to the set $D \cup(K-E)$. In reference to the function we refer to, we merely extend the function continuously from $T_{1} \cup T_{2} \cup T_{3}$ to the complement of $E$ rather than extending it just to the open upper half plane. Then we restrict the function to the open upper half plane.

Let $f$ be the function defined in $D$ which has the above properties. Then

$$
\Pi(f, \zeta)=\Pi_{x}(f, \zeta)=\varnothing \quad(\zeta \in E) .
$$

Since $f$ can be continuously extended to $D \cup(K-E)$, we have that

$$
\Pi(f, \zeta)=\Pi_{x}(f, \zeta) \neq \varnothing \quad(\zeta \in K-E) .
$$

The definition of $B \Pi(f, \zeta)$ implies that if $B \Pi\left(f, \zeta_{0}\right)$ is empty for some point $\zeta_{0} \in K$, then $\Pi(f, \zeta)$ is empty for every point in some nondegenerate subarc of $K$. But this is impossible since $K-E$ is dense in $K$ and $\Pi(f, \zeta)$ is nonempty for $\zeta \in K-E$. Thus $B \Pi(f, \zeta)$ is nonempty for every $\zeta \in K$, and similarly $B \Pi_{x}(f, \zeta)$ is nonempty for every $\zeta \in K$. Consequently

$$
\Pi(f, \zeta) \neq B \Pi(f, \zeta) \quad \text { and } \quad \Pi_{x}(f, \zeta) \neq B \Pi_{x}(f, \zeta) \quad(\zeta \in E) .
$$

\section{REFERENCES}

1. F. Bagemihl, G. Piranian and G. S. Young, Intersections of cluster sets, Bul. Inst. Politehn. Iaşi (N.S.) 5 (9) (1959), no. 3-4, 29-34. MR 22 \#8118.

2. F. Bagemihl, Some results and problems concerning chordal principal cluster sets, Nagoya Math. J. 29 (1967), 7-18. MR 35 \#4425. 
3. C. L. Belna, Intersections of arc-cluster sets for meromorphic functions, Nagoya Math. J. 40 (1970), 213-221.

4. E. F. Collingwood and A. J. Lohwater, The theory of cluster sets, Cambridge Tracts in Math. and Math. Phys., no. 56, Cambridge Univ. Press, Cambridge, 1966. MR 38 \#325.

5. P. Lappan and D. C. Rung, Normal functions and non-tangential boundary arcs, Canad. J. Math. 18 (1966), 256-264. MR 33 \#7553.

6. K. Noshiro, Cluster sets, Ergebnisse der Mathematik und ihrer Grenzgebiete, N.F., Heft 28, Springer-Verlag, Berlin, 1960. MR 24 \#A3295.

7. G. T. Whyburn, Analytic topology, Amer. Math. Soc. Colloq. Publ., vol. 28, Amer. Math. Soc., Providence, R. I., 1963. MR 32 \#425.

Department of Mathematics, Bowling Green State University, Bowling Green, ОнІо 43403 